\title{
Predicted scattering of sound by diffuse hydrothermal vent plumes at mid-ocean ridges
}

\author{
Timothy F. Duda \\ Applied Ocean Physics and Engineering Department, Woods Hole Oceanographic Institution, Woods Hole, \\ Massachusetts 02543 \\ D. Andrew Trivett \\ Trivett Technologies, Inc., Mahone Bay, Nova Scotia BOJ 2EO, Canada
}

(Received 12 April 1996; revised 6 January 1997; accepted 1 September 1997)

\begin{abstract}
Amplitude and phase fluctuations of monochromatic acoustic signals traveling through diffuse mid-ocean ridge hydrothermal vent plumes are modeled using existing theory in an attempt to find suitable frequencies and path lengths for plume monitoring. Weak-scattering solutions are evaluated numerically, with model parameters adjusted to match observed plume characteristics. Constraints required for weak-scattering solutions to be valid can be met for transmission ranges of 500-2000 $\mathrm{m}$ and frequencies of $20-80 \mathrm{kHz}$. Therefore, because fluid structure and scattering strength are more closely linked for weak scattering than for stronger scattering, inversion for fluid statistical properties may be possible, enabling diffuse vent monitoring. Such monitoring would be subject to geometric assumptions such as transmission entirely within a statistically homogeneous plume. Performance-limiting phase fluctuations have also been computed for a $13-17 \mathrm{kHz}$ geodetic survey system. (C) 1998 Acoustical Society of America. [S0001-4966(98)01801-3]
\end{abstract}

PACS numbers: 43.30.Pc, 43.30.Re [JHM]

\section{INTRODUCTION}

Fluctuations that develop as radiation passes through a randomly inhomogeneous medium can provide a view of the medium characteristics. Since sound propagates freely through water, it has been the wave of choice for this type of remote sensing in the ocean. The usefulness of the view obtained, and the reliability of its interpretation, is dependent on the both the nature of the inhomogeneities and the regime of the acoustic scattering.

Propagation modeling in the Rytov weak-scattering regime (Fante, 1975; Strohbehn, 1978; Tatarskii, 1971) is used here to investigate the potential of a forward-scattered acoustic measurement of mid-ocean ridge hydrothermal venting. This type of measurement would involve transmission from a tethered source to a tethered receiver. The signals would be fluctuations of phase and amplitude, to be associated in an inverse fashion with thermal fluctuation statistics along the propagation path. Details of the modeling technique are described in two previous publications (Duda, 1991; Duda et al., 1988). Parameters of the modeling are adjusted to be appropriate for diffuse vent fluid using data from a field study at the Juan de Fuca Ridge (Trivett and Williams, 1994). Since a forward model is required for inverse estimation of fluid properties, a primary aspect of this work is ascertaining that the Rytov model is applicable to experimentally realistic frequencies and propagation distances. The Rytov model is accurate for situations having acoustic fluctuations below some threshold, but breaks down for increased fluctuation strength.

Diffuse vent effluent is only slightly warmer than the deep ocean water and generally resides in a bottom-hugging plume (Trivett, 1994) rather than in a buoyant plume wafting in the current. An acoustic remote sensing scheme may be possible for these plumes since they are trapped at a known vertical location near the bottom. Remote sensing may be beneficial for diffuse plume monitoring because of the inherent volumetric averaging. For example, a volumetric measure may determine whether point measurements (such as with thermometers) are spatially representative, or may more accurately characterize a larger volume. Also, some success may be achieved at circumventing the so-called "gopherhole" problem of outflow sensing, where the total output of a hydrothermal area may not be measurable with sensors at a few vent openings because of transient vent birth or mortality (Tivey, 1991).

In principle, if the acoustic fluctuations are not too large, weak-scattering theory can be used to relate aspects of the scattered field to the intervening medium. The weakscattering behavior is an important requirement for fluid characterization because it implies a close link between medium fluctuation statistics and acoustic fluctuation statistics. This close relation is not true for stronger scattering which exhibits "saturation" (Ishimaru, 1978), a convergence of statistics. Many underwater propagation regimes exhibit either very weak or very strong scattering, such as vertical propagation away from boundaries (weak) and horizontal propagation through strong turbulence in a mixed layer or a coastal region (strong). The weak-scattering cases may not provide adequate signal for fluid characterization, a problem as severe as saturation from strong scattering. On the other hand, diffuse vent flows in otherwise essentially thermally homogeneous deep-ocean water masses may be a situation of adequate scattering, but not strong scattering, where acoustics can be modeled, understood, and inverted for measurement purposes. Other constraints on fluid characterization exist, such as homogeneity and isotropy of refractive index variations within the water, but these complications of 
inverse-based remote sensing can be considered and dealt with only after weak scattering has been verified.

The physics behind this possibility of acoustic remote sensing are easily understood. The development of a sound pulse moving through a strongly heterogeneous medium from a simple to a complex pattern, as range increases, eventually provides multiple arrivals at great range. At short ranges multipath may not exist, but the propagation processes which eventually lead to multipath cause pulse distortions. Properties of these distortions (which can be thought of as incipient multipath) are dependent on properties of the medium. We suppose that these distortions, manifested as phase and amplitude fluctuations, can be measured and used to monitor rms thermal gradients at deep-ocean vent sites.

The obvious analog of the long-range ocean tomographic mapping (Munk et al., 1995), i.e., the use of pulse timing to map an anomalously warm plume, is not feasible near hydrothermal vent areas unless acoustic sources and receivers can be firmly fixed to the bottom. This is because timing signals developed over the short ranges would be very small, and any motions would overwhelm the signals (Trivett, 1991). Because the pressure gradient in the nearly isothermal deep ocean causes sound to refract upward, only instruments on promontories could avoid the shadowing effect. However, the statistics of fluctuations in phase and amplitude which develop can be measured with tethered devices, without consideration of overall timing, and related to the sound-speed (temperature) statistics of the intervening medium. Such statistical remote sensing has been used in the atmosphere and the solar wind (Coles and Kaufman, 1978; Yeh and Liu, 1982).

The goals of this short study are to determine frequencies and path lengths where weak scattering describes the propagation, and to determine the sensitivity of intensity fluctuation statistics to plume turbulence properties. Additionally, phase statistics of $15-\mathrm{kHz}$ weakly scattered signals will be calculated for the purpose of providing signalprocessing bounds on deep-ocean ridge acoustic geodetic systems (Spiess and Hildebrand, 1995).

\section{WEAK SCATTERING}

The Rytov weak-scattering model, also called the method of smooth perturbations, quantifies forward scattering through a random medium. This is a single-scattering approximation, with the field at the receiver expressed as an integral over range of scattered fields. The incident field at each range is composed of the field as it would exist in the absence of medium fluctuations. Statistical properties of fields in planes transverse to the propagation direction can be expressed in terms of statistical properties of the intervening medium. The expressions for spatial spectra of phase and amplitude perturbations can be integrated to give variances, assuming spatial variability can be substituted for ensemble averaging, an assumption consistent with homogeneity and stationarity of environmental fluctuation statistics, and also consistent with the spectral description. A suitable expression from previous work is the double integral (Duda, 1991; Duda et al., 1988)

$$
\begin{aligned}
\Theta(m)= & 2 \pi R q^{2} \int_{0}^{1} d s \frac{m}{s^{2}} \int_{-\infty}^{\infty} d l F\left(0, k_{y}=\frac{l m}{s}, k_{z}=\frac{m}{s}\right) \\
& \times G\left[\frac{m^{2} R_{f}^{2}}{4 \pi s}(1-s)\left(l^{2}+1\right)\right] .
\end{aligned}
$$

The notation is consistent with the cited publications. Transmission is in the $x$ direction over a range $R . F(\mathbf{k})$ is the vector spectrum of sound-speed fluctuations, so that soundspeed variance $\left\langle\mu^{2}\right\rangle=\int d^{3} \mathbf{k} F(\mathbf{k})$, with $\mathbf{k}=\left(k_{x}, \mathbf{k}_{\mathbf{T}}\right)$ $=\left(k_{x}, k_{y}, k_{z}\right)$ and $k=|\mathbf{k}| . \Theta$ is the wave number power spectrum of either log amplitude or phase, $m$ is wave number in the $z$-direction transverse to the propagation path at range $R$ (vertical as written), $k_{z}$ and $k_{y}$ are transverse wave numbers of inhomogeneities in the intervening medium, $q$ is a reference acoustic wave number in an isovelocity medium, $s$ $=x / R$ is a normalized range coordinate, and $l=k_{y} s / \mathrm{m}$ is a scaled lateral wave number. The Fresnel radius is $R_{f}$ $=(2 \pi R / q)^{1 / 2}$. The function $G$ is $\operatorname{sine}^{2}$ if we consider $\log$ amplitude, cosine $^{2}$ if phase.

Expression (1) for the acoustic fluctuation spatial spectrum is derived from the expression for the acoustic field under the aforementioned scattering assumption, from which a covariance expression is developed. The spectrum follows directly from the covariance via Fourier transform. Expression (1) is useful for modeling scattering from turbulence because it involves the spatial spectrum of the thermal fluctuations, which are related in a known manner to fundamental properties of the turbulent field such as turbulent energy production rate and thermal variance dissipation rate $\chi$ (Batchelor, 1959).

Computations will include integration of log-amplitude spectra $\Theta$ over wave number $m$, giving log-amplitude variance, which is $1 / 4$ times $\log$-intensity variance, since logintensity $\iota=2 \ln A=\ln (I /\langle I\rangle)$. Since $\iota+1 \approx I /\langle I\rangle$ in the region of validity, log-intensity variance is equal to the scintillation index $\sigma_{I}^{2}=\left(\left\langle I^{2}\right\rangle-\langle I\rangle^{2}\right) /\langle I\rangle^{2}$, a measure of scattering, which is a normalized intensity variance. The limits of applicability of Rytov theory are often quoted to be $\sigma_{I}^{2}<0.3$ (Fante, 1975), but phase fluctuation calculations may be valid beyond this to $\sigma_{I}^{2}<0.7$ (Ewart and Reynolds, 1984).

\section{SPECTRA OF SOUND-SPEED STRUCTURE}

As in the previous work, the medium is modeled as a homogeneous volume of isotropic sound-speed fluctuations. These are modeled with an isotropic inertial-convective scalar subrange spectrum (Batchelor, 1953; Tatarskii, 1971). A high-pass filter with outer scale $k_{t}$ is used to confine the subrange to $k_{t}<k$, and a diffusive roll-off $R_{b}$ from the Batchelor spectrum is included, giving

$$
F_{m}(\mathbf{k})=\frac{\phi_{t}}{\left(k^{2}+k_{t}^{2}\right)^{11 / 6}} \frac{k^{2}}{k^{2}+k_{t}^{2}} R_{b}(k) .
$$

The scalar quantity $\phi_{t}$ is a spectral amplitude parameter. $F_{m}$ indicates model $F$. This model is most sensible in a weakly stratified region, such as a surface mixed layer or a bottom boundary layer. It can also be realistic in stratified parts of the ocean, but stratification generally confines the subrange 
to scales of $1 \mathrm{~m}$ and less (Dillon, 1982). Sound-speed structure at scales smaller than the viscous cutoff scale $l_{c}$ $=\left(\nu^{3} \epsilon^{-1}\right)^{1 / 4}$, tens of centimeters or less, is poorly modeled by $F_{m}$ ( $\nu$ is the kinematic viscosity and $\epsilon$ is the rate of dissipation of kinetic energy), but the precise form in the rolloff region is not relevant since microstructure of scales $R_{f}$ or larger contribute dominantly to acoustic fluctuations in the weak fluctuation regime. The simulations here have $R_{f}$ ranging from $12.2 \mathrm{~m}(20-\mathrm{kHz}, 2-\mathrm{km}$ range $)$ to $2.1 \mathrm{~m}(80-\mathrm{kHz}$, $250-\mathrm{m}$ range), and most of the scattering is caused by the largest modeled scales, determined by $k_{t}=0.1 \mathrm{rad} \mathrm{m}^{-1}$.

Vector spectra $F$ which are isotropic, as are $F_{m}$, can be integrated over spherical shells to give wave number magnitude spectra (also called three-dimensional spectra), $E(k)$ $=2 \pi k^{2} F(k)$. One-dimensional spectra $D\left(k_{x}\right)$ for any direction $x$, which contain aliased contributions from high wave numbers, are related by the expression $E(k)=-k$ $\times(d D(k) / d k)$ (Tennekes and Lumley, 1972). In the next section it is shown that measured $D(k)$ from diffuse plume fluid can be used to find parameters of $F_{m}(k)$ which are best matched to diffuse plumes.

\section{MODEL MEDIUM CONSTRAINTS FROM FIELD MEASUREMENTS}

One-dimensional spectra $D(k)$ can be computed from the temperature records of Trivett and Williams. During 1990, two bottom-resting, 5-m tall tripods with vertical arrays of thermistors and three-axis current meters were placed on the Southern Juan de Fuca Ridge during the VENTS'90 field study. Four tethered arrays of thermistors were also deployed. Representative data from one tripod are used here. The 45-m tall tethered arrays exhibited a clear distinction between variable (in plume) and essentially isothermal (out of plume) periods, each of many hours duration, encouraging this study of an averaging acoustic measurement tool. The tripod records were closer to the mixing influences of the rough volcanic terrain and had lower peak variances than the higher thermistors, so we will examine model spectra of magnitude equal to and greater than those consistent with the tripod measurements.

Spectra $D\left(k_{x}\right)$ of sound-speed fluctuations recorded from fluid advecting past tripod BS3 are shown in Fig. 1. These are recorded within $3 \mathrm{~m}$ of the bottom. The temporal sampling is once per minute, the mean velocity past the array is $3.5 \mathrm{~cm} \mathrm{~s}^{-1}$, and anisotropic fluctuations having horizontal scales of up to tens of meters are included. The spatial spectra of Fig. 1 are calculated from temperature time-series assuming Taylor's frozen field hypothesis and the above stated velocity. A loosely fit $k^{-5 / 3}$ inertial-subrange spectrum is also shown.

Properly scaled acoustic fluctuation predictions would result from models having spectra $F_{m}$ sharing common spectra $E$ with the measured $D$ of Fig. 1. This is because $E$ can be computed from either $D$ or $F$ using relations of the previous section. Figures 2 and 3 show $E$ computed from the higher-level $D$ of Fig. 1, along with $E$ spectra from the loosely fit inertial-subrange $D$. The slope of the measured $E$ changes at wavelength of roughly $40 \mathrm{~m}$, possibly indicating a change in dynamics at that scale. Figures 2 and 3 also show

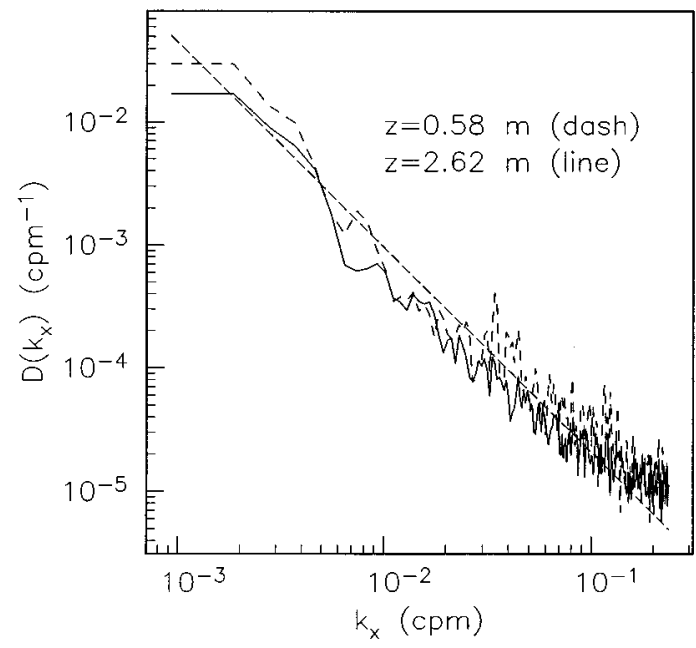

FIG. 1. The one-dimensional spectra $D\left(k_{x}\right)$ of sound speed ( $c$ ) from temperature $(T)$ records at levels 1 and 4 of the tripod BS3 are shown (Trivett and Williams, 1994). The heights above the bottom are indicated. The sampling rate was $2 \mathrm{~Hz}$, but these data are averaged and recorded at 1-min intervals, so the Nyquist period is $120 \mathrm{~s}$. The conversion to wave number uses the mean speed measured at the same tripod, $0.035 \mathrm{~m} / \mathrm{s}$, giving a Nyquist wave number of about $4.2 \mathrm{~m}$. The local $d c / d T$ coefficient is $4.37 \mathrm{~m} \mathrm{~s}^{-1}{ }^{\circ} \mathrm{C}^{-1}$. The straight dashed line shows a fitted $k^{-5 / 3}$ inertial subrange spectrum.

three example $E(k)$ derived from $F_{m}(k)$ used for propagation modeling. An important physical parameter is the thermal dissipation rate $\chi$, which in the absence of salinity variations can be computed from the spectrum in this way:

$$
\chi=2 D_{t} \alpha^{2} \int_{0}^{\infty} k^{2} E(k) d k
$$

where $D_{t}$ is the molecular diffusivity of heat, $\alpha=d T / d c, T$ is temperature, and $c$ is sound speed. A more general defini-

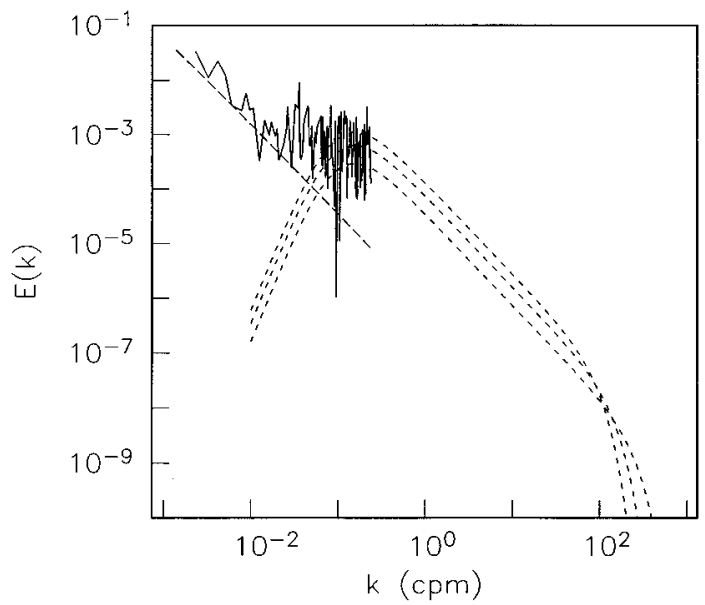

FIG. 2. Spectra $E$ computed from the one-dimensional spectra of Fig. 1 are compared with $E$ computed from three model spectra $F_{m}$. The curves at lower wave number are from the $k^{-5 / 3}$ power-law example $D$ (straight dashed line) and the more elevated of the two measured $D$ (solid line). Each of the three model curves (dashed curves) has thermal dissipation rate $\chi$ $=10^{-9}{ }^{\circ} \mathrm{K}^{2} \mathrm{~s}^{-1}$, with energy dissipation equal to $10^{-9}, 10^{-10}$, and 2 $\times 10^{-11} \mathrm{~W} \mathrm{~kg}^{-1}$. The model height at the peak is inversely related to $\epsilon$ and to bandwidth. 


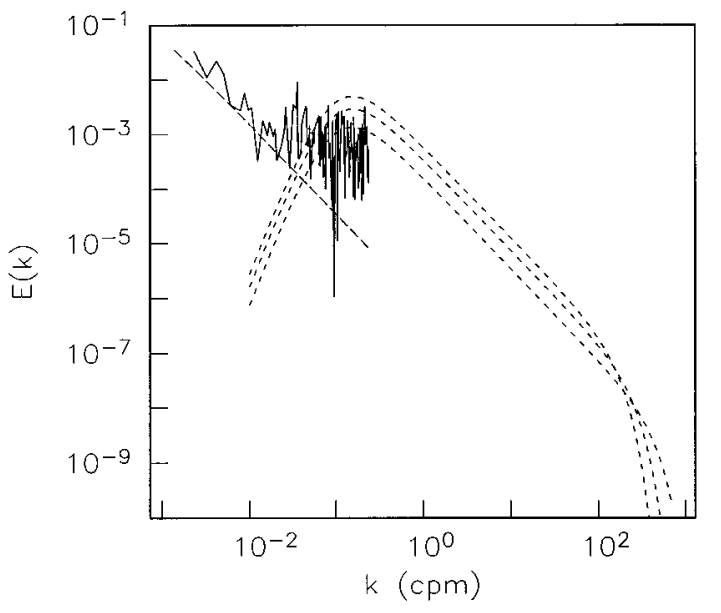

FIG. 3. Similar to Fig. 2, but the model $E$ use $\chi=10^{-8}{ }^{\circ} \mathrm{K}^{2} \mathrm{~s}^{-1}$, and energy dissipations $10^{-8}, 10^{-9}$, and $2 \times 10^{-10} \mathrm{~W} \mathrm{~kg}^{-1}$. These models spectra have significantly more variance at $R_{f}$ scales (meters) than those of Fig. 2.

tion of $\chi$ is in terms of the temperature gradient variance. The parameter $\chi$ is fixed for the family of model curves in each figure; energy dissipation rate $\epsilon$ varies.

Some details of $F_{m}(k)$ are useful to keep in mind. Since $F_{m}$ are band limited, for a given $\chi$ the spectral magnitude at a given scale varies inversely with the turbulence spatial bandwidth, which is governed at high wave number by Batchelor's theory and increases with increasing $\epsilon$ (Batchelor, 1959). The ratio $M=\chi / \epsilon$ is a scaled version of mixing efficiency in the steady-state situation, because $\chi$ is proportional to thermal variance elimination (mixing) and $\epsilon$ is related to source energy in the steady state. Since the outer scale is fixed at $k_{t}=0.1 \mathrm{rad} \mathrm{m}^{-1}$, the maximum variance occurs at 5-10 m wavelength. Substantial fluctuations exist to wavelengths as long as $15 \mathrm{~m}$. Fluctuations drop off rapidly away from the peak wavelength. The outer scale is chosen to loosely conform with our best ideas of plume dynamics (Trivett, 1994). It is consistent with a weakly stratified lateral plume many tens of meters in height above the seafloor, entraining fluid, with eddies at all scales up to the plume height.

\section{WEAK SCATTERING MODEL RESULTS}

Models $F_{m}$ [given by Eq. (2)] consistent with measured $D$ are chosen for evaluation of Eq. (1), with subsequent integration of $\Theta(m)$ (spectrum of either log intensity or phase) over all $m$ to give $\sigma_{I}^{2}$ or the variance of phase. Based on comparisons resembling Figs. 2 and $3, F_{m}$ having $\chi$ of 4 $\times 10^{-8}, 10^{-8}, 4 \times 10^{-9}$, and $10^{-9}{ }^{\circ} \mathrm{K}^{2} \mathrm{~s}^{-1}$ were used. These had variable energy dissipation (or turbulent production), and thus variable spatial bandwidth, plus a variable mixing efficiency $M$. The resultant sound-speed spectra bracket the experimental spectra in the band from $10 \mathrm{~m}$ to 2 $\mathrm{m}$ wavelength. These are the smallest measured scales and the largest modeled scales. Their effect will dominate the modeled acoustic fluctuations, which should be compared to acoustic observations after longer period fluctuations (such as from tidal effects) are filtered out.
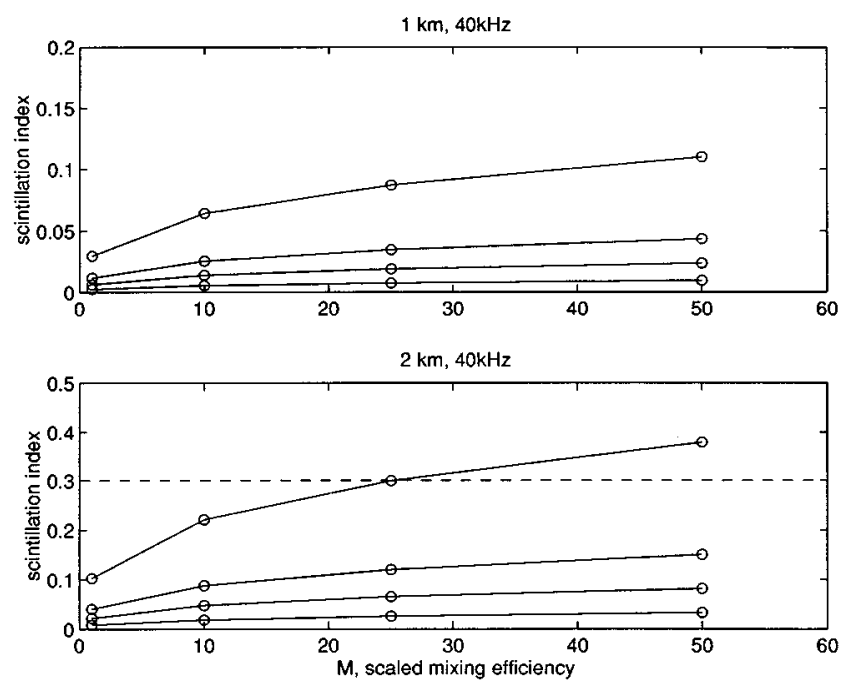

FIG. 4. Scintillation index $\left(\sigma_{I}^{2}\right)$ results for the $40-\mathrm{kHz}$ frequency, 1- and 2-km range $\left(R_{f}=6.1,8.7 \mathrm{~m}\right)$ are plotted versus scaled mixing efficiency $M$. Thermal dissipation $\chi$ is fixed for each curve in the family, with $\chi=4$ $\times 10^{-8}{ }^{\circ} \mathrm{K}^{2} \mathrm{~s}^{-1}$ for the upper curve, descending to $10^{-8}, 4 \times 10^{-9}$, and $10^{-9}$. The scaled mixing efficiency is the ratio $\chi / \epsilon$, units ${ }^{\circ} \mathrm{K}^{2} \mathrm{~s}^{-1}$ over $\mathrm{W} \mathrm{kg}^{-1}$. It can be seen that $\sigma_{I}^{2}$ is below or near the weak-scattering limit (dashed line) for many situations.

Figures 4 through 6 , showing $\sigma_{I}^{2}$ for $40-80 \mathrm{kHz}$ at $1-2$ $\mathrm{km}$ range, show $\sigma_{I}^{2}$ exceeding 0.3 for the highest of the models, but well below it in most cases. This is a promising result for plume monitoring. Figures 7 and 8 show weaker fluctuations for $20-\mathrm{kHz}$ acoustics and short-range $80 \mathrm{kHz}$.

The figures show that $\sigma_{I}^{2}$ is a weak function of $M$, as expected, compared with the sensitivity to $\chi$. Factor of 40 changes of $\chi$ at fixed $M$ have about triple the effect of a factor of 50 change in $M$ at fixed $\chi$. This is expected, since $M$ changes vary the turbulent bandwidth only a small percentage, with small changes in spectral height near $R_{f}$ and thus in scattering.
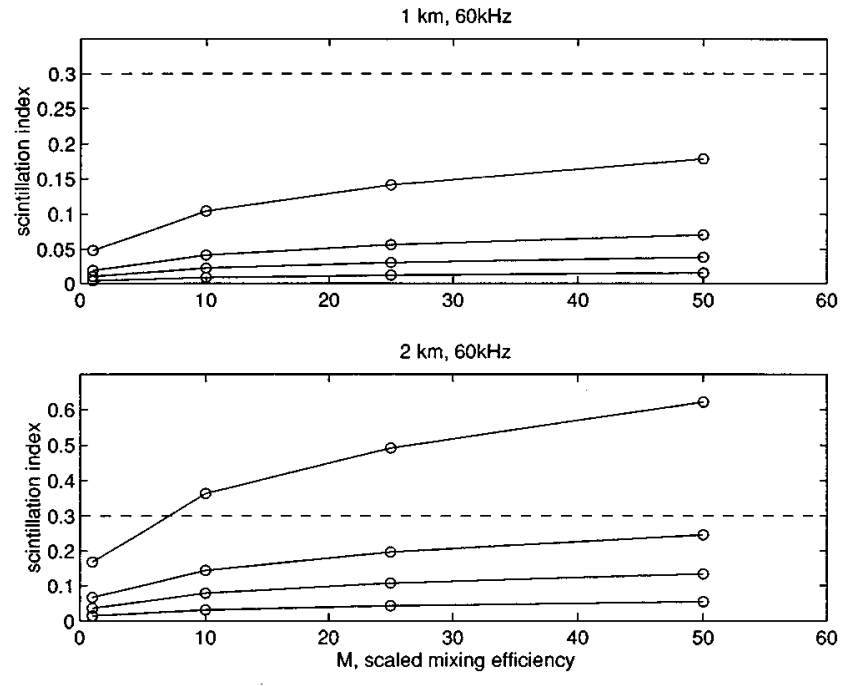

FIG. 5. Scintillation index results for the $60-\mathrm{kHz}$ frequency, 1 -and $2-\mathrm{km}$ range $\left(R_{f}=5 \cdot 0,7.1 \mathrm{~m}\right)$, which are slightly greater than the $40-\mathrm{kHz}$ results of Fig. 4. The parameter $\chi$ is fixed for each curve, having the same values as in Fig. 4. 

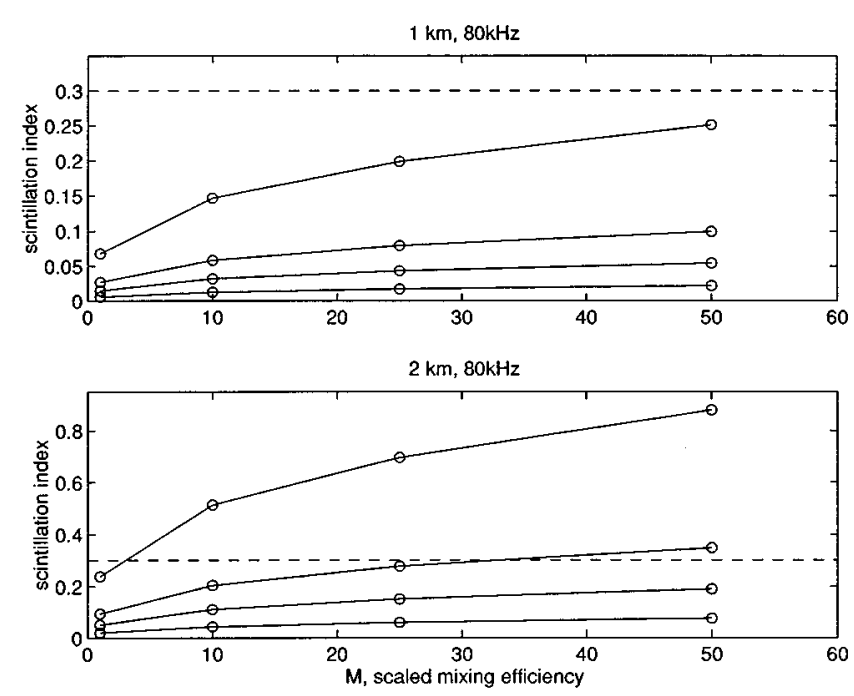

FIG. 6. Scintillation index results for the $80-\mathrm{kHz}$ frequency, 1 and $2 \mathrm{~km}$ $\left(R_{f}=4.3,6.1 \mathrm{~m}\right)$ are shown. The index greatly exceeds the weak scattering limits of applicability $(0.3)$ for $\chi=4 \times 10^{-8}$, at most energy dissipations for the $2-\mathrm{km}$ range, but not for the 1-km range. $\chi$ for each curve is as in Fig. 4.

The curves in each of the panels show that multiple values of $\chi$ and $M$ will give identical $\sigma_{I}^{2}$ values. This nonuniqueness means that either additional observations, or better constraints on plume structures and physics, may be required for total accuracy of monitoring.

An indirect-path seafloor geodetic system (Spiess and Hildebrand, 1995) using 13-17 kHz frequency-sweep pulse compression has been designed. The indirect-path system measures the acoustic travel time between a deep-towed platform and individual bottom-mounted transceivers in order to infer their positions with respect to one another. To provide estimates of how diffuse venting would degrade the signals, rms phase (travel time) fluctuation is estimated for $15 \mathrm{kHz}$, and the three ranges 1,2 , and $4 \mathrm{~km}$. Figure 9 shows that the rms phase (arrival time) fluctuations are distributed from a
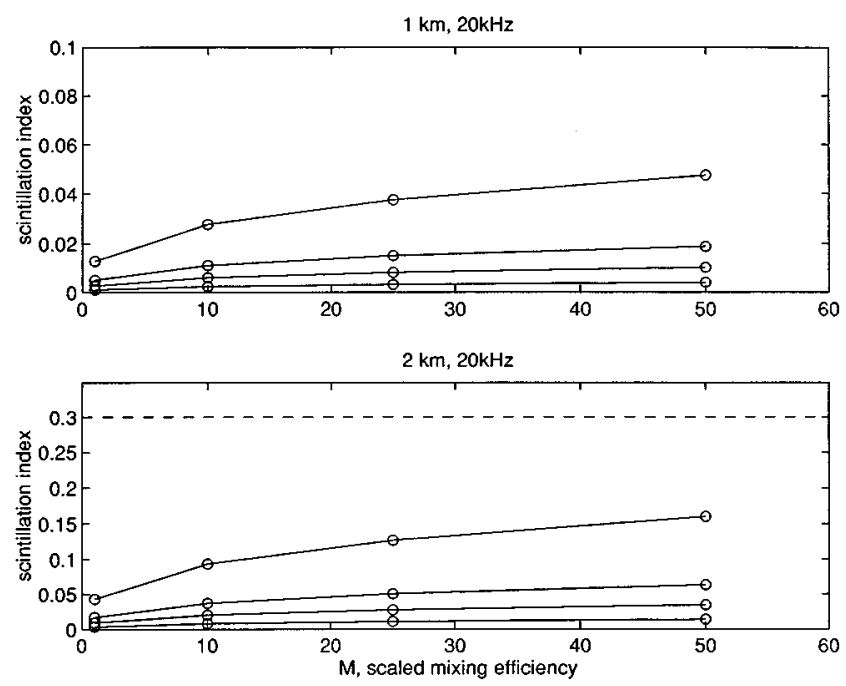

FIG. 7. Scintillation indices for the $20-\mathrm{kHz}$ frequency, 1 and $2 \mathrm{~km}\left(R_{f}\right.$ $=8.7,12.2 \mathrm{~m}$ ), are quite weak, especially for $1 \mathrm{~km} . \chi$ for each curve is as listed in Fig. 4.
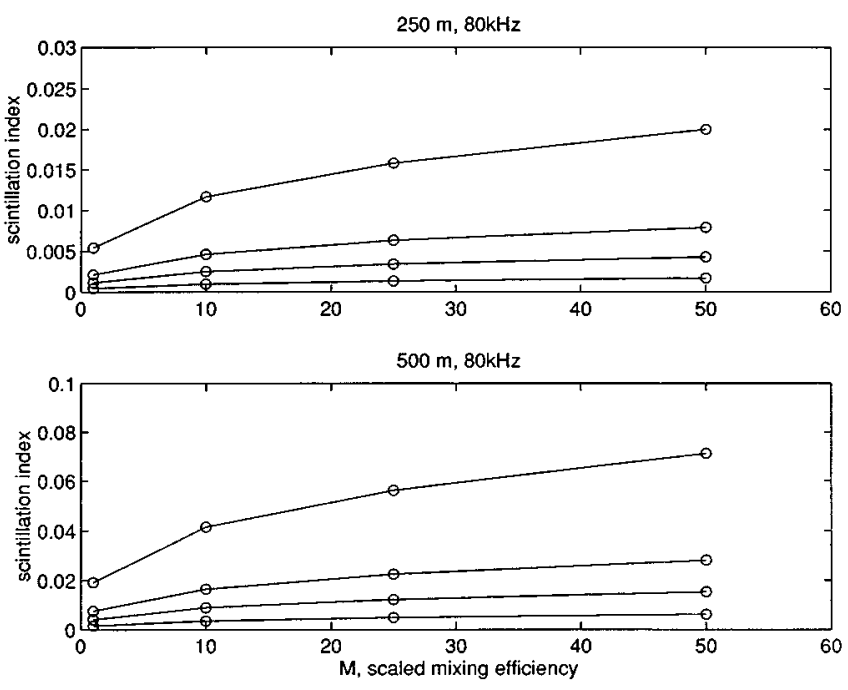

FIG. 8. Scintillation indices for the $80-\mathrm{kHz}$ frequency, $250-\mathrm{m}$ and $500-\mathrm{m}$ range $\left(R_{f}=2.2,3.1 \mathrm{~m}\right)$, are smaller than those of Figs. 4-7 for longer ranges. There is less propagation range over which phase interference patterns can develop, resulting in very small fluctuations. $\chi$ for each curve is as in Fig. 4.

few degrees, for low mixing efficiency, to maxima near 40 degrees for propagation through $4 \mathrm{~km}$ of weak plume heterogeneities, and near 20 degrees for $1-\mathrm{km}$ propagation. Converting to length units, 25 degrees of a $15-\mathrm{kHz}$ wave is equal to $7 \mathrm{~mm}$. This is near the desired performance levels of the systems, which are intended to measure possible variability of seafloor spreading rates which average a few centimeters per year.

\section{SUMMARY}

Our modeled acoustic propagation through isotropic, homogeneous random sound-speed (temperature) structures show that the weak-scattering criteria can be met for a selection of ranges and frequencies. The structures are consistent with observations taken near an ocean ridge hydrothermal vent. When these criteria are met, changes in intensity variance can be related to changes in the medium with some reliability, assuming some knowledge of the physics of the fluctuation medium.

Strong, measurable scintillations, but not too strong to invalidate the modeling, are predicted for $40-80 \mathrm{kHz}$ signals over ranges of 1-2 km. Many effects are scalable and other ranges and frequencies will give similar results.

The scale length of the inhomogeneities which have the dominant effect is a function of both the acoustic frequency and range. The ranges and frequencies quoted above are most sensitive to structures of 4-9 $\mathrm{m}$ wavelength. Temperature variability at such scales is enhanced in vent plumes (Trivett and Williams, 1994) but is typically weak in the deep ocean, implying scattering to be a good diagnostic tool for verification of plume existence.

The solution of the forward problem does not necessarily put the inverse problem within grasp. Nonuniqueness, or multiple possible natural conditions consistent with acoustic observations, is a potential difficulty. More detailed monitoring would be possible when future measurements and better 

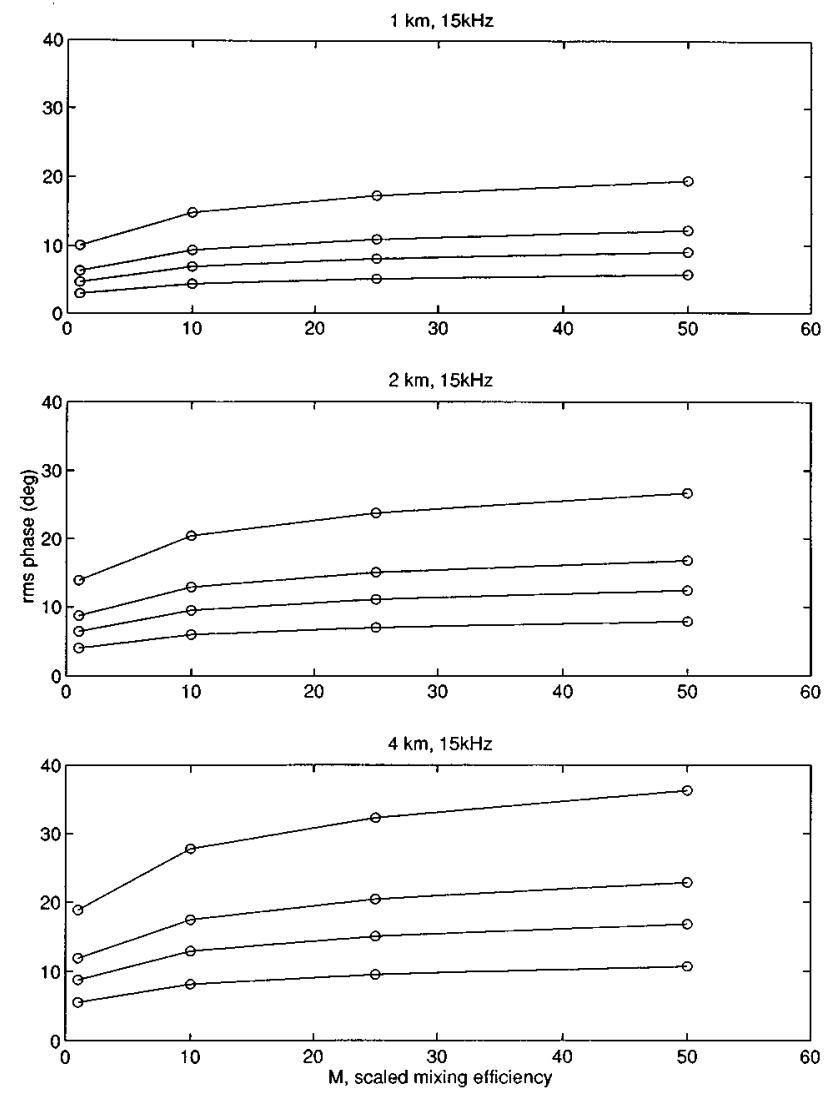

FIG. 9. rms phase fluctuation estimates for $15 \mathrm{kHz}$ at the 1-, 2-, and 4-km range $\left(R_{f}=10,14\right.$, and $\left.20 \mathrm{~m}\right)$ are shown. These are functions of $M$ and thermal dissipation rate $\chi$, as in Figs. $4-8$. The parameter $\chi$ is fixed for each curve; the values are as listed in Fig. 4 . The acoustic wavelength is $10 \mathrm{~cm}$, so the indicated fluctuations approach travel times equivalent to a centimeter of range.

plume understanding allow more restrictive propagation modeling and unique invertibility. However, plume monitoring should be possible initially, even without full verification of plume dynamics. Signals should exist as long as the plumes are weakly mixing, and not fully mixed, so that the larger meter-scale gradients remain.

Phase fluctuations were estimated for deep-sea acoustic geodetic systems signals, and these show that fluctuations of the same magnitude of the expected signals may exist in the worst case scenario of propagation fully through an effluent plume. In this case, a more elaborate survey with higher redundancy and greater data quantity may be required.

\section{ACKNOWLEDGMENTS}

This work was supported by the Woods Hole Oceanographic Institution with research funds provided by the Mellon Foundation. This is WHOI Contribution No. 9256.

Batchelor, G. K. (1953). The Theory of Homogeneous Turbulence (Cambridge U.P., New York).

Batchelor, G. K. (1959). "Small-scale variation of convected quantities like temperature in a turbulent fluid," J. Fluid Mech. 5, 113-133.

Coles, W. A., and Kaufman, J. J. (1978). "Solar wind estimation from multi-station IPS,"' Radio Sci. 13, 591-597.

Dillon, T. M. (1982). "Vertical overturns: A comparison of Thorpe and Ozmidov length scales," J. Geophys. Res. 87, 9601-9613.

Duda, T. F. (1991). "Modeling weak fluctuations of undersea telemetry signals," IEEE J. Ocean Eng. 16, 3-11.

Duda, T. F., Flatté, S. M., and Creamer, D. B. (1988). "Modelling meterscale acoustic intensity fluctuations from oceanic fine structure and microstructure," J. Geophys. Res. 93, 5130-5142.

Ewart, T. E., and Reynolds, S. A. (1984). "The mid-ocean acoustic transmission experiment: MATE," J. Acoust. Soc. Am. 75, 785-802.

Fante, R. L. (1975). "Electromagnetic beam propagation in turbulent media," Proc. IEEE 63, 1669-1692.

Ishimaru, A. (1978). Wave Propagation and Scattering in Random Media (Academic, New York), Vol. 2.

Munk, W., Worcester, P., and Wunsch, C. (1995). Ocean Acoustic Tomography (Cambridge U.P., New York).

Spiess, F. N., and Hildebrand, J. A. (1995). "Employing geodesy to study temporal variability at a mid-ocean ridge," EOS Trans. Am. Geophys. Union 76, 451,455.

Strohbehn, J. W. (1978). "Modern theories in the propagation of optical waves in a turbulent medium," in Laser Beam Propagation in the Atmosphere, edited by J. W. Strohbehn (Springer-Verlag, New York).

Tatarskii, V. I. (1971). The Effects of the Turbulent Atmosphere on Wave Propagation, National Technical Information Service, TT-68-50464, Springfield, VA.

Tennekes, H., and Lumley, J. L. (1972). A First Course in Turbulence (MIT, Cambridge, MA).

Tivey, M. K. (1991). Private communication.

Trivett, D. A. (1991). "Diffuse flow from hydrothermal vents," Mass. Inst. Technol./Woods Hole Oceanogr. Inst. Joint Program in Oceanogr. Eng., Sc.D. Dissertation, Woods Hole, MA.

Trivett, D. A. (1994). "Effluent from diffuse hydrothermal venting 1. A simple model of plumes from diffuse hydrothermal sources,' J. Geophys. Res. 99, 18 403-18 415.

Trivett, D. A., and Williams III, A. J. (1994). "Effluent from diffuse hydrothermal venting 2. Measurement of plumes from diffuse hydrothermal vents at the southern Juan de Fuca Ridge,' J. Geophys. Res. 99, 18 41718432 .

Yeh, K. C., and Liu, C. H. (1982). "Radio wave scintillations in the ionosphere," Proc. IEEE 70, 324-360. 